\title{
Estimativa DA ÁREA FoliaR DE Synedrellopsis grisebachii UsANDo MÉTOdo NÃo DESTRUTIVO ${ }^{1}$
}

\author{
Synedrellopsis grisebachii Foliar Area Estimate Using a Non-Destructive Method \\ CARVALHO, L.B. ${ }^{2}$, BIANCO, M.S. ${ }^{3}$ e BIANCO, S. ${ }^{4}$
}

\begin{abstract}
RESUMO - A área foliar é uma das principais características usadas para avaliar o crescimento vegetal. O objetivo desta pesquisa foi determinar uma equação matemática para estimar a área foliar de Synedrellopsis grisebachii - uma importante planta daninha no Brasil - a partir de dimensões lineares dos limbos foliares. Duzentas folhas foram medidas em comprimento (C), largura máxima (L) e área foliar real (AF). Os dados de AF e CxL foram submetidos à análise de regressão linear, determinando-se uma equação matemática para estimar a área foliar da espécie. A correlação entre os valores de área foliar real e estimada foi significativa. Portanto, a área foliar de S. grisebachii pode ser estimada satisfatoriamente pela equação: $\mathrm{AF}=0,730829(\mathrm{C} \times \mathrm{L})$.
\end{abstract}

Palavras-chave: agriãozinho, biometria, crescimento, equação matemática.

\begin{abstract}
Leaf area is one of the main characteristics used to evaluate plant growth. This work aimed to determine a mathematical equation to estimate the leaf area of Synedrellopsis grisebachii, an important weed in Brazil, using the linear measures of the leaf blade. Length (L), width (W) and real leaf area (LA)of two hundred leaves were measured. $L A$ and $L \times W$ data were submitted to linear regression analysis, adjusting a mathematical equation to estimate the leaf area of the species. The correlation between the real and estimated leaf area values was significant. Thus, the leaf area of S. grisebachii may be estimated through the equation $L A=0.730829(L \times W)$.
\end{abstract}

Keywords: straggler daisy, biometry, growth, mathematical equation.

\section{INTRODUÇÃO}

Synedrellopsis grisebachii, popularmente conhecida por agriãozinho, é uma planta daninha nativa da América do Sul, frequentemente encontrada nas regiões Centro-Sul e Centro-Oeste do Brasil. É uma planta perene, prostrada, herbácea, de caules ramificados, glabros e com enraizamento nos nós, que se reproduz por sementes, rizomas e enraizamento dos ramos; apresenta considerável desenvolvimento vegetativo, ocorrendo desde solos arenosos e friáveis até solos argilosos (Kissmann \& Groth, 1999; Lorenzi, 2000).
Estudos básicos a respeito de reprodução, crescimento, desenvolvimento, exigência nutricional, respostas aos sistemas de controle e outros aspectos são necessários para melhor compreensão da biologia e escolha de métodos de manejo de espécies daninhas (Bianco et al., 2008a,b). Na maioria desses estudos, o conhecimento da área foliar é fundamental, pois é uma das características mais importantes na avaliação do crescimento vegetal (Portela, 1999; Bhatt \& Chanda, 2003; Bianco et al., 2008a,b; 2009), além de auxiliar na compreensão de relações de interferência entre plantas daninhas e cultivadas, uma vez

Recebido para publicação em 13.4.2011 e aprovado em 17.6.2011.

2 Pós-Doutorando, Bolsista FAPESP, Dep. de Biologia Aplicada à Agropecuária, FCAV/UNESP, Jaboticabal, <agrolbcarvalho@gmail.com>; ${ }^{3}$ Mestrando em Produção Vegetal, Bolsista CAPES, Dep. de Produção Vegetal, FCAV/UNESP, Jaboticabal; ${ }^{4}$ Prof. Livre Docente, Dep. Biologia Aplicada à Agropecuária, FCAV/UNESP, Jaboticabal, <sbianco@fcav.unesp.br> . 
que a área foliar é uma das características mais rapidamente afetadas pela interferência das plantas daninhas.

A estimativa da área foliar de plantas pode ser feita por diversos métodos, sendo os não destrutivos mais interessantes, pois não há necessidade da coleta da planta, ou de parte dela, para as medições. Entre os métodos não destrutivos mais utilizados está a determinação de equações matemáticas relacionando as dimensões lineares da folha (Bianco et al., 2007a,b, 2008a,b, 2009; Duarte et al., 2009).

A estimativa da área foliar por meio de equações matemáticas tem sido utilizada com boa precisão, sendo um método fácil e rápido, tornando-se importante para avaliar o crescimento das plantas em campo (Bianco et al., 2007a). Equações de regressão, relacionando área foliar real com características dimensionais lineares das folhas, têm sido utilizadas com sucesso para estimativa da área foliar de diversas plantas daninhas (Bianco et al., 2008a,b, 2009) e também em diversas plantas cultivadas (Queiroga et al., 2003; Araújo et al., 2005; Monteiro et al., 2005; Souza et al., 2005; Cittadini \& Peri, 2006; Alves et al., 2009; Busato et al., 2009; Posse et al., 2009).

A hipótese de trabalho foi de que a área foliar de S. grisebachii pode ser expressa por uma equação matemática, o que facilita a determinação dessa característica importante na avaliação do crescimento vegetal diretamente no campo, sem destruição do material vegetal. O objetivo desta pesquisa foi determinar uma equação matemática que possa estimar adequadamente a área foliar de S. grisebachii a partir da relação entre as dimensões lineares dos limbos foliares, para utilização como método não destrutivo.

\section{MATERIAL E MÉTODOS}

As folhas de S. grisebachii utilizadas neste estudo foram coletadas em meados do mês de maio de 2009, em diferentes ecossistemas agrícolas, como cana-de-açúcar, culturas anuais (milho e soja), áreas de olericultura e pomares de frutíferas, buscando amostrar plantas nas diferentes condições ecológicas. Foram coletadas 200 folhas de S. grisebachii, em todas as posições da planta e em plantas com diferentes estádios de desenvolvimento, desde que não apresentassem deformações oriundas de fatores externos, como pragas, doenças, granizo, etc.

Em cada local de amostragem, 10 a 20 folhas de diferentes plantas foram coletadas; em seguida, rapidamente foi feita, em laboratório, a determinação do comprimento do limbo foliar ao longo da nervura principal (C) e da largura máxima do limbo foliar perpendicular à nervura principal (L), de cada folha, através de medições manuais com régua graduada em milímetros. Posteriormente, a área real de cada folha (AF) foi determinada, com a utilização de um medidor eletrônico de área foliar (Portable Area Meter, Licor Mod. LI 3000A).

Para o estudo da relação entre a área do limbo foliar e as dimensões dos limbos foliares, foi realizada a estimativa da equação linear $Y$ $=a . X$, em que $Y$ expressa o valor da área do limbo foliar; $a$, um parâmetro de reta que corresponde ao percentual de equivalência entre as áreas foliares; e $X$, o produto do comprimento $(C)$ pela largura (L). Essa equação foi escolhida, pois Bianco et al. (2007a,b, $2008 \mathrm{a}, \mathrm{b})$, entre outros, concluíram que a equação linear que passa pela origem é satisfatória para estimar a área foliar, além de ser aquela de melhor aplicação prática.

Assim, os dados de área foliar e comprimento x largura dos limbos foliares de S. grisebachii foram submetidos à análise de variância pelo teste $\mathrm{F}(\mathrm{p}<0,05)$ e à análise de regressão pelo modelo citado anteriormente.

Complementarmente, para averiguar a correlação entre os valores reais e estimados de área foliar da espécie através da equação matemática determinada, procedeu-se ao teste de correlação de Spearman-Rank $(\mathrm{p}<0,05)$.

Todas as análises estatísticas foram processadas por meio do programa computacional Statistica 6.0 (StatSoft, 2001).

\section{RESULTADOS E DISCUSSÃO}

S. grisebachii apresentou valores médios de $2,09 \mathrm{~cm}, 1,10 \mathrm{~cm}$ e $1,64 \mathrm{~cm}^{2}$ de comprimento, largura e área foliar, respectivamente, com o comprimento representando $1,9 \mathrm{vez}$ a 
largura da folha (Tabela 1). A maior porcentagem da área foliar de $S$. grisebachii esteve contida na faixa de 1,01 a 1,50 $\mathrm{cm}^{2}$, e $90 \%$ da área foliar da espécie esteve na faixa de 1,01 a 2,50 $\mathrm{cm}^{2}$ (Tabela 2). Segundo Kissmann \& Groth (1999), as folhas de S. grisebachii são curto-pecioladas, de limbo ovalado com ápice arredondado ou agudo, apresentando, em média, 2,00 cm de comprimento por $1,50 \mathrm{~cm}$ de largura.

O parâmetro $a$ da equação linear ajustada aos dados de área foliar real, em relação ao comprimento x largura dos limbos foliares de S. grisebachii, apresentou o valor de 0,730829, cujo intervalo de confiança aceitável esteve entre 0,717503 e 0,744154 (Tabela 3); a análise de variância da regressão evidenciou diferença entre os dados analisados $(p<0,05)$ (Tabela 4). Portanto, evidencia-se que a área foliar de S. grisebachii aumenta linearmente com o aumento de comprimento x largura dos limbos foliares, e $81 \%$ dos dados puderam ser representados pela equação ajustada (Figura 1). Nesse caso, propõe-se que a área do limbo foliar seja proporcional a um retângulo $\mathrm{CxL}$, utilizando-se de um fator de correção $a$ para o cálculo da área foliar da planta. Assim, quanto mais próximo à unidade for o valor do fator de correção, mais proporcional a um retângulo será a morfologia do limbo foliar da planta.

Entre as plantas daninhas de "folhaslargas" já estudadas, Ipomoea hederifolia (Bianco et al., 2007a), Sida cordifolia e Sida rhombifolia (Bianco et al., 2008b) apresentaram maiores valores do parâmetro a que S. grisebachii, enquanto para Ipomoea nil (Bianco et al., 2007a), Leonotis nepetaefolia (Bianco et al., 2007b) e Ageratum conyzoides (Bianco et al., 2008a) os valores observados foram menores. Isso evidencia que C. glaberrima, I. hederifolia,
S. cordifolia e S. rhombifolia apresentam a morfologia do limbo foliar mais proporcional a um retângulo que $S$. grisebachii, enquanto em A. conyzoides, I. nil, L. nepetaefolia e T. procumbens essa proporcionalidade é menor.

Tabela 1 - Valores máximo, mínimo e médio do comprimento ao longo da nervura central, da largura máxima e da área foliar real de Synedrellopsis grisebachii

\begin{tabular}{|l|c|c|c|}
\hline \multicolumn{1}{|c|}{ Característica } & Maior valor & Menor valor & Valor médio \\
\hline Comprimento $(\mathrm{cm})$ & 3,00 & 1,10 & 2,09 \\
\hline Largura $(\mathrm{cm})$ & 1,90 & 0,70 & 1,10 \\
\hline Área foliar $\left(\mathrm{cm}^{2}\right)$ & 3,70 & 0,94 & 1,64 \\
\hline
\end{tabular}

Tabela 2 - Porcentagem de limbos foliares distribuídos em diferentes faixas de tamanho relativas à área foliar de Synedrellopsis grisebachii

\begin{tabular}{|c|c|}
\hline Área foliar $\left(\mathrm{cm}^{2}\right)$ & Porcentagem \\
\hline $0,51-1,00$ & 3,50 \\
\hline $1,01-1,50$ & 47,00 \\
\hline $1,51-2,00$ & 29,50 \\
\hline $2,01-2,50$ & 13,50 \\
\hline $2,51-3,00$ & 4,00 \\
\hline$>3,10$ & 2,50 \\
\hline
\end{tabular}

Tabela 3 - Resultado da análise de regressão, pelo modelo y = $a . \mathrm{x}$, dos dados reais de área foliar e comprimento $\mathrm{x}$ largura dos limbos foliares de Synedrellopsis grisebachii

\begin{tabular}{|c|c|c|c|}
\hline$a$ & Erro-padrão & $\begin{array}{c}\text { Limite de } \\
\text { confiança } \\
\text { inferior }\end{array}$ & $\begin{array}{c}\text { Limite de } \\
\text { confiança } \\
\text { superior }\end{array}$ \\
\hline 0,730829 & 0,006758 & 0,717503 & 0,744154 \\
\hline
\end{tabular}

Tabela 4 - Resultado da análise de variância pelo teste $\mathrm{F}(\mathrm{p}<0,05)$ da regressão dos dados reais de área foliar vs comprimento $\mathrm{x}$ largura dos limbos foliares de Synedrellopsis grisebachii

\begin{tabular}{|l|r|r|r|r|c|}
\hline \multicolumn{1}{|c|}{ Causa de variação } & SQR & GL & QMR & F & p \\
\hline Regressão & 581,19 & 1 & 581,19 & $11.696,52$ & $<0,001$ \\
\hline Resíduos & 9,89 & 199 & 0,05 & & \\
\hline Total & 591,08 & 200 & & & \\
\hline Total corrigido & 51,39 & 199 & & & \\
\hline Regressão $v s$ Total corrigido & 581,19 & 1 & 581,19 & $2.250,57$ & $<0,001$ \\
\hline
\end{tabular}




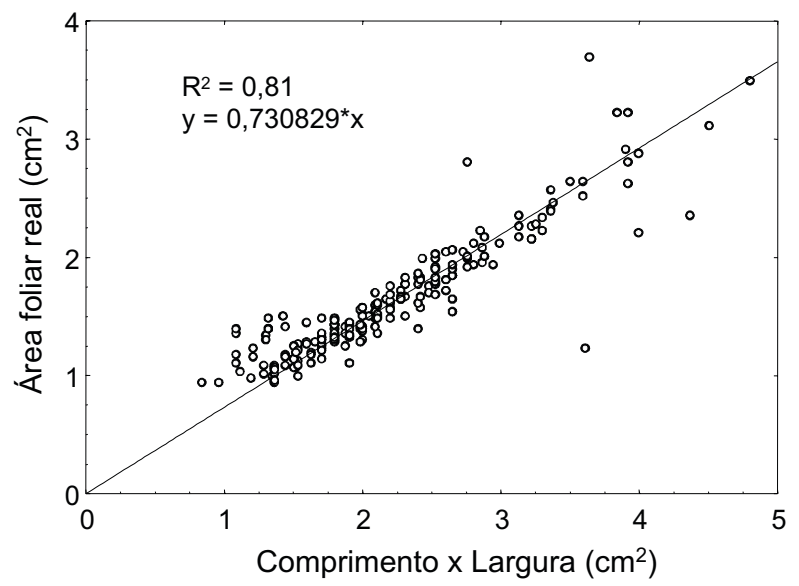

Figura 1 - Relação linear entre a área foliar real e o comprimento x largura dos limbos foliares de Synedrellopsis grisebachii.

O teste de correlação aplicado sobre os valores reais e estimados de área do limbo foliar de $S$. grisebachii foi significativo $(\mathrm{p}<0,05)$, com $92 \%$ de correlação entre os dados analisados (Tabela 5), evidenciando, assim, segundo Bianco et al. (2008b), que a equação ajustada representa significativamente a área do limbo foliar da espécie.

Segundo Gamiely et al. (1991), o uso de equações de regressão para estimar a área foliar de plantas é um método simples, rápido, preciso e confiável, que permite acompanhar o crescimento e a expansão foliar de uma mesma planta, até o final do ciclo ou do experimento, sem precisar coletar o material vegetal. Esse método elimina a necessidade de destruição da folha, o que afetaria o desenvolvimento da planta e tornaria equivocada a avaliação do experimento.

Assim, a área do limbo foliar de S. grisebachii pode ser estimada satisfatoriamente pela equação $\mathrm{Y}=0,730829(\mathrm{CxL})$, em que Y expressa a área foliar estimada e $\mathrm{CxL}$ representa o produto do comprimento ao longo da nervura principal pela largura máxima do limbo foliar.

Tabela 5 - Resultado da análise de correlação pelo teste SpermanRank $(\mathrm{p}<0,05)$ sobre os dados reais e estimados de área foliar de Synedrellopsis grisebachii

\begin{tabular}{|c|c|c|c|c|}
\hline Variáveis & $\begin{array}{c}\text { Valor } \\
\text { válido }\end{array}$ & R-Spearman & $\mathrm{t}(\mathrm{N}-2)$ & $\mathrm{p}$ \\
\hline $\begin{array}{c}\text { Área foliar real } v s \\
\text { área foliar estimada }\end{array}$ & 200 & 0,92 & 33,12 & $<0,001$ \\
\hline
\end{tabular}

\section{LITERATURA CITADA}

ALVES, W. W. A. et al. Área foliar do algodoeiro irrigado com água residuária adubado com nitrogênio e fósforo. $\mathbf{R}$. Verde, v. 4, n. 1, p. $41-46,2009$

ARAÚJO, E. C. E. et al. Estimativa da área foliar da mangueira (Mangifera indica L.) cvs. Tommy Atkins e Haden, utilizando dimensões lineares. R. Bras. Frutic., v. 27, n. 2, p. 308-309, 2005.

BHATT, M.; CHANDA, S. V. Prediction of leaf area in Phaseolus vulgaris by non-destructive method. Bulgarian $\mathbf{J}$. Plant Physiol., v. 29, n. 1, p. 96-100, 2003.

BIANCO, S. et al. Caracterização da área foliar de Merremia aegyptia. Planta Daninha, v. 27, p. 941-945, 2009 (número especial).

BIANCO, S. et al. Estimativa da área foliar de Ipomoea hederifolia e Ipomoea nil Roth. usando dimensões lineares do limbo foliar. Planta Daninha, v. 25, n. 2, p. 325-329, 2007a.

BIANCO, S.; BIANCO, M. S.; CARVALHO, L. B. Estimativa da área foliar de Leonotis nepetifolia (L.) W.T. Aiton usando dimensões lineares do limbo foliar. Ci. Cult., v. 2 , n. 1 , p. $27-31,2007 \mathrm{~b}$.

BIANCO, S.; BIANCO, M. S.; CARVALHO, L. B. Estimativa da área foliar de Ageratum conyzoides usando dimensões lineares do limbo foliar. Acta Sci. Agron., v. 30, n. 4, p. 519-523, $2008 \mathrm{a}$.

BIANCO, S.; CARVALHO, L. B.; BIANCO, M. S Estimativa da área foliar de Sida cordifolia e Sida rhombifolia usando dimensões lineares do limbo foliar. Planta Daninha, v. 26, n. 4 , p. $807-813,2008$ b.

BUSATO, C. et al. Estimativa da área foliar da cultivar de batata Cupido. Hortic. Bras., v. 27, n. 2, p. 3570-3573, 2009

CITTADINI, E. D.; PERI, P. L. Estimation of leaf area in sweet cherry using a non-destructive method. R. Investig. Agropec., v. 35, n. 1, p. 143-150, 2006.

DUARTE, D. J. et al. Estimativa da área foliar de Euphorbia heterophylla. Planta Daninha, v. 27, n. 3, p. 527-531, 2009.

GAMIELY, S. et al. A rapid and nondestructive method for estimating leaf area of onions. HortScience, v. 26, n. 2 , p. 206,1991

KISSMANN, K. G.; GROTH, D. Plantas infestantes e nocivas. 2.ed. São Paulo: BASF, 1999. Tomo II. 798 p.

LORENZI, H. Plantas daninhas do Brasil: terrestres, aquáticas, parasitas e tóxicas. 3.ed. Nova Odessa: IPEF, 2000. $640 \mathrm{p}$. 
MONTEIRO, J. E. B. A. et al. Estimação da área foliar do algodoeiro por meio de dimensões e massa das folhas.

Bragantia, v. 64, n. 1, p. 15-24, 2005.

PORTELA, J. A. Planteo de modelos para la estimación del área foliar en plantas de ajo (Allium sativum $\mathrm{L}$.) tipo clonal «blanco». Hortic. Argentina, v. 18, n. 1, p. 5-10, 1999.

POSSE, R. P. et al. Total leaf area of papaya trees estimated by a nondestructive method. Sci. Agric., v. 66, n. 4, p. $462-466,2009$.
QUEIROGA, J. L. et al. Estimativa da área foliar de feijãovagem (Phaseolus vulgaris L.) por meio da largura máxima do folíolo central. Hortic. Bras., v. 21, n. 1, p. 64-68, 2003.

SOUSA, E. F. et al. Estimating the total leaf area of the green dwarf coconut tree (Cocos nucifera L.). Sci. Agric., v. 62, n. 6, p. 597-600, 2005.

STATSOFT, INC. Statistica: data analysis software system. Version 6.0. Tulsa: 2001. 